\title{
XALIA: rationale and design of a non-interventional study of rivaroxaban compared with standard therapy for initial and long-term anticoagulation in deep vein thrombosis
}

\author{
Walter Ageno ${ }^{1}$, Lorenzo G Mantovani², Sylvia Haas ${ }^{3}$, Reinhold Kreutz ${ }^{4}$, Verena Haupt ${ }^{5}$, Jonas Schneider ${ }^{6}$
} and Alexander GG Turpie 7* $^{*}$

\begin{abstract}
Venous thromboembolism (VTE), comprising deep vein thrombosis (DVT) and pulmonary embolism, poses a substantial clinical risk, and the incidence of these thrombotic-related diseases remains high. Anticoagulation aims to prevent thrombus extension and reduce the risk of recurrent events, particularly fatal pulmonary embolism. In EINSTEIN DVT, rivaroxaban was non-inferior to enoxaparin/vitamin $\mathrm{K}$ antagonists for the reduction of recurrent VTE, with a similar safety profile and a net clinical benefit. EINSTEIN EXT investigated patients receiving long-term treatment in whom there was no clear decision about continuing or stopping anticoagulation; rivaroxaban was superior to placebo in the reduction of recurrent VTE, showing an acceptable benefit-risk balance. Rivaroxaban has the potential to replace standard therapy, usually parenteral low molecular weight heparin overlapping with and followed by a vitamin $\mathrm{K}$ antagonist, for the treatment of acute symptomatic DVT and the secondary prevention of VTE. As the use of rivaroxaban for DVT treatment increases in clinical practice, a fundamental understanding of its clinical benefits in everyday patient care is essential. XALIA (XArelto for Long-term and Initial Anticoagulation in venous thromboembolism) is a multicentre, prospective, non-interventional, observational study investigating the effectiveness and safety of a single-drug approach with rivaroxaban compared with standard therapy in patients with DVT. The study cohort will include approximately 4800 patients ( $\geq 18$ years old) with objectively confirmed acute DVT who will be treated for a period of $\geq 3$ months. The primary outcomes will be the incidence of treatment-emergent adverse events (primarily major bleeding), symptomatic recurrent venous thromboembolic events and all-cause mortality. Secondary outcomes include: major cardiovascular events; patient-reported treatment satisfaction and adherence; healthcare resource utilization; reasons for drug switching or interruption of treatment; and adverse events. XALIA will follow an international cohort of patients in more than 20 European countries, and others including Israel and Canada. The first patient was enrolled in June 2012, with results expected in 2015. It is anticipated that XALIA will provide important information on the treatment of DVT in a heterogeneous, unselected patient population in a real-world setting and provide important supplementary information to that obtained from the EINSTEIN DVT phase III study.
\end{abstract}

Keywords: Deep vein thrombosis, Outcomes, Real-world experience, Rivaroxaban

\footnotetext{
* Correspondence: turpiea@mcmaster.ca

${ }^{7}$ Department of Medicine, Hamilton Health Sciences, General Division, 237

Barton Street East, Hamilton, Ontario L8L 2X2, Canada

Full list of author information is available at the end of the article
} 


\section{Introduction}

Acute venous thromboembolism (VTE), which comprises deep vein thrombosis (DVT) and pulmonary embolism $(\mathrm{PE})$, is the third most common cardiovascular disorder [1]. VTE occurs with an incidence of 1-2 cases per 1000 people in the general population and the risk increases with age $[2,3]$. Treatment of VTE involves an initial phase, in which the key imperative is to prevent thrombus extension, improve acute symptoms and reduce the risk of early PE [4]. The current standard treatment for acute VTE is overlapping anticoagulation with a parenteral agent (such as low molecular weight heparin [LMWH] ) and a vitamin K antagonist (VKA) [5]. VKAs may be given over a period of months, years, or even indefinitely, to reduce the risk of recurrent events. Guidelines on the duration of anticoagulant therapy for VTE, including those published by the American College of Chest Physicians (ACCP), recommend at least 3 months' treatment after a provoked or unprovoked event [5].

Anticoagulation with LMWH is rapid and effective, but the need for daily subcutaneous injections can be challenging. Heparin-induced thrombocytopenia may be a risk with LMWH use, with an incidence of $\leq 1 \%$ in medical and postoperative patients [6]. The limitations of VKAs in routine clinical practice are well documented: treatment with these agents requires regular monitoring to ensure patients remain within the therapeutic range (international normalized ratio 2.0-3.0), and a wide range of drug-drug and drug-food interactions may influence therapeutic levels of anticoagulation. Initial adjustment of VKA dosing in the first weeks of therapy is particularly challenging and in some cases is not easy to achieve [7]. Furthermore, the use of a dual-drug approach with LMWH and VKAs can be both complex and burdensome for patients and healthcare providers.

Direct oral Factor Xa inhibitors, such as apixaban, edoxaban and rivaroxaban, and the direct oral thrombin inhibitor dabigatran, have recently been investigated in phase III clinical trials for the treatment and secondary prevention of DVT and PE [8-15]. Rivaroxaban and apixaban have both been studied as a single-drug approach, whereas edoxaban and dabigatran employed an initial course of parenteral heparin therapy before commencing therapy with the anticoagulant being investigated. Data show that these agents are effective and well tolerated; however, it is not known whether these findings are directly applicable to patients in a real-world setting. As the use of the direct oral anticoagulants in clinical practice increases, a fundamental understanding of their efficacy and safety in patients with VTE treated in routine care is essential. XALIA (XArelto for Long-term and Initial Anticoagulation in venous thromboembolism) will investigate the effectiveness of a single-drug approach with rivaroxaban compared with standard dual-drug therapy with LMWH/VKAs in the real-world treatment of patients with DVT.

\section{Rivaroxaban}

Rivaroxaban is a direct oral Factor Xa inhibitor with a high oral bioavailability $(\geq 80 \%)[16]$ and predictable pharmacokinetics and pharmacodynamics $[17,18]$; these characteristics provide the basis for use of this agent without the need for routine coagulation monitoring. The onset of action of rivaroxaban, i.e. its anti-Factor Xa activity, has been shown to be as fast as that of the LMWH enoxaparin [19]. Peak plasma concentrations of rivaroxaban are achieved within 2-4 hours and it has a mean half-life of approximately 9 hours (5-9 hours in young individuals and 11-13 hours in the elderly) $[17,18]$. Rivaroxaban is associated with few drug-drug [20-22] or drug-food interactions [23]. However, rivaroxaban doses of $15 \mathrm{mg}$ and $20 \mathrm{mg}$ should be taken with food to maintain high oral bioavailability [23].

XALIA is being conducted in response to a request made by the European Medicines Agency (EMA) to evaluate rivaroxaban for the treatment of acute DVT after the completion of the large phase III EINSTEIN DVT study. The EINSTEIN phase III clinical programme investigated the use of rivaroxaban as a single-drug approach in contrast to the current dual-drug paradigm of enoxaparin/VKAs [8,9]. EINSTEIN DVT was a randomized, open-label, event-driven, non-inferiority trial that investigated the efficacy and safety of oral rivaroxaban ( $15 \mathrm{mg}$ twice daily for 21 days followed by $20 \mathrm{mg}$ once daily) compared with subcutaneous enoxaparin $(1 \mathrm{mg} / \mathrm{kg}$ body weight twice daily for at least 5 days) overlapping with and followed by a VKA (warfarin or acenocoumarol, started within 48 hours after randomization), in patients with acute, symptomatic, objectively confirmed proximal DVT without PE. Treatment duration was at the discretion of the attending physician and was planned for 3, 6 or 12 months [8]. In EINSTEIN DVT, rivaroxaban was shown to be noninferior to enoxaparin/VKA for the reduction of recurrent VTE (Table 1) [8]. Rates of first major or non-major clinically relevant bleeding (the primary safety outcome) were similar in patients receiving rivaroxaban compared with patients receiving enoxaparin/VKA. In addition, rivaroxaban was associated with a significantly improved net clinical benefit (defined as the composite of symptomatic, recurrent VTE and major bleeding), compared with enoxaparin/VKA (Table 1) [8]. The consideration of patient-reported outcomes is becoming increasingly important when evaluating the overall effectiveness of direct oral anticoagulants. It is worth noting that an EINSTEIN DVT subanalysis showed that rivaroxaban was associated with improved treatment satisfaction compared with enoxaparin/VKA therapy in patients with DVT [24]. 
Table 1 Efficacy and safety outcomes of the phase III EINSTEIN DVT study

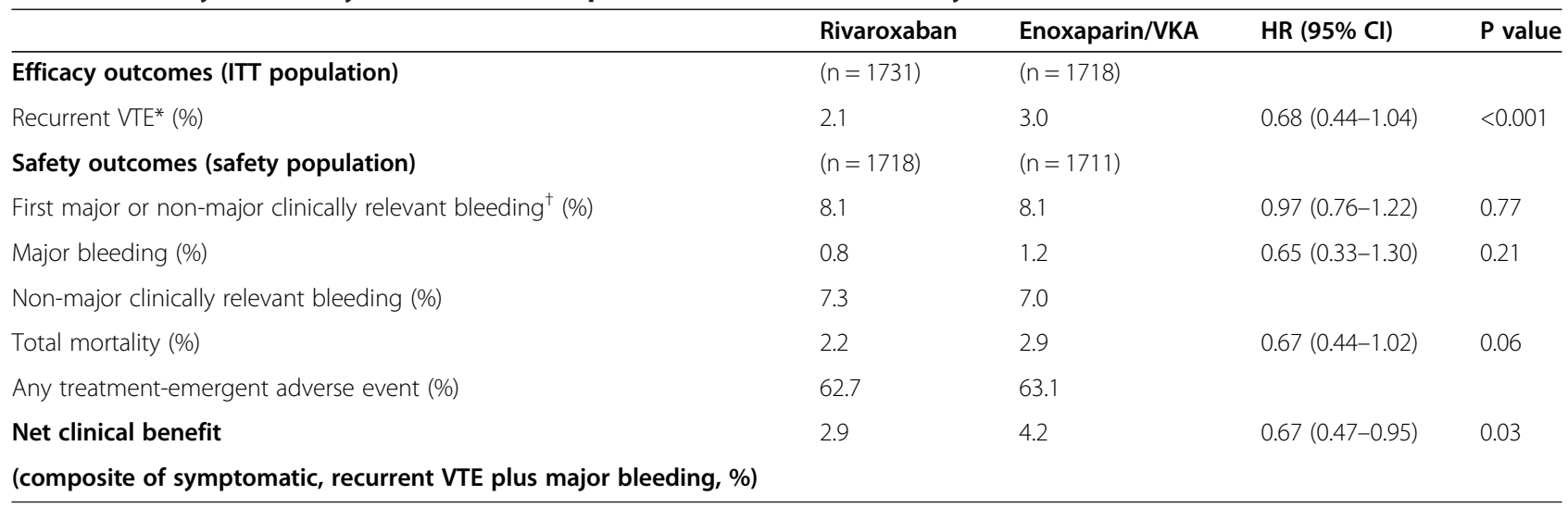

*Primary efficacy outcome; ${ }^{\dagger}$ principal safety outcome.

$\mathrm{Cl}$, confidence interval; DVT, deep vein thrombosis; HR, hazard ratio; ITT, intention to treat; PE, pulmonary embolism; VKA, vitamin K antagonist; VTE, venous thromboembolism.

Among the factors noted were a reduction in patientreported anticoagulation burden and an increase in convenience [24]. It would, therefore, be desirable to confirm the influence of rivaroxaban treatment on patient lives in routine care.

The EINSTEIN PE study investigated rivaroxaban in patients with acute, symptomatic $\mathrm{PE}$, and also showed that it was non-inferior to enoxaparin/VKA, with a $51 \%$ reduction in the incidence of major bleeding [9]. The EINSTEIN EXT study investigated rivaroxaban in patients receiving long-term treatment in whom there was no clear decision about continuing or stopping anticoagulation; rivaroxaban was superior to placebo in the reduction of recurrent VTE, and showed an acceptable benefit-risk balance [8].

Rivaroxaban is the first direct oral anticoagulant to be approved for the treatment of DVT and PE and secondary prevention of VTE in the European Union, US and Canada. XALIA is a multicentre, prospective, non-interventional, observational study taking place across more than 20 European countries, and other countries including Israel and Canada. XALIA will assess whether the results obtained with rivaroxaban in real-world clinical practice in patients with objectively confirmed, acute DVT and an indication to receive anticoagulation for at least 3 months are consistent with those seen in the phase III EINSTEIN DVT study.

The main objective of the study is to evaluate the safety of rivaroxaban in routine clinical practice for the treatment of acute symptomatic DVT and prevention of recurrent VTE by examining the incidence of treatment-emergent adverse events: major bleeding, symptomatic recurrent venous thromboembolic events and all-cause mortality.

\section{XALIA study design}

The study cohort consists of patients with acute DVT treated with rivaroxaban or current standard therapy (initial treatment with unfractionated heparin, LMWH or fondaparinux, which may overlap with and be followed by an oral VKA) for a period of $\geq 3$ months. The study duration will be 12 months from the final date of patient enrolment, but the treatment duration for each patient will be determined solely by the attending physician and does not depend on the initial intended treatment duration or the study duration. The first patient was enrolled in June 2012 and the end of study is expected in 2015.

\section{Administrative organization}

Non-interventional post-authorization safety studies are recommended for review by Ethics Committees, and the study/sponsor sought approval from an independent Ethics Committee or Institutional Review Board in all countries where such procedures were in place.

An external expert scientific Steering Committee will give support regarding study objectives, study conduct and analysis. An independent Adjudication Committee blinded to the treatment group will adjudicate events. Members of the Steering Committee may also serve on the Adjudication Committee; however, no sponsor representatives will serve on the Adjudication Committee. The study is registered at clinicaltrials.gov (NCT01619007).

\section{Centre and patient selection}

Recruitment of patients will be conducted by investigators at selected study centres in Europe and other regions in which rivaroxaban is approved. Enrolment is planned for approximately 4800 patients with approximately equal numbers in each arm. Patients can be enrolled immediately after a decision about the choice of anticoagulant therapy has been made, but no randomization will be undertaken. Inclusion and exclusion criteria are summarized in Table 2. 
Patients who are $\geq 18$ years old, with a diagnosis of objectively confirmed, acute DVT and an indication to receive anticoagulation for $\geq 3$ months will be included. If anticoagulation is administered for a shorter period than planned, patients will remain in the study.

\section{Study outcomes}

Primary study outcomes are the incidence of treatmentemergent adverse events, namely major bleeding, symptomatic recurrent venous thromboembolic events and all-cause mortality. Major bleeding is defined as overt bleeding associated with a fall in haemoglobin of $\geq 2 \mathrm{~g} / \mathrm{dl}$; or a transfusion of $\geq 2$ units of packed red blood cells or whole blood; or bleeding at a critical site (intracranial, intraspinal, intraocular, pericardial, intra-articular, intramuscular with compartment syndrome, retroperitoneal); or death. Secondary outcomes include: major cardiovascular events; patient-reported treatment satisfaction and adherence; healthcare resource utilization; reasons for drug switching or interruption of treatment; and adverse events, including serious adverse events. Data on patients with renal impairment treated for acute DVT within the terms of the local product information will also be collected and analysed. The Adjudication Committee, blinded to the treatment group, will adjudicate bleeding events; recurrent VTE and other thromboembolic events; major cardiovascular events; and cause of death. Local objective diagnosis of symptomatic recurrent venous thromboembolic events is a prespecified criterion for event adjudication.

\section{Drug administration}

The type, dose and duration of drug therapy are at the sole discretion of the attending physician. Enrolment will only be considered after the treatment decision has been made. Patients who commence treatment with rivaroxaban, or those who initially receive treatment with heparin/fondaparinux within 48 hours of enrolment, can be included in the rivaroxaban cohort. Patients in the standard-of-care cohort are those receiving other recommended pharmacological treatments for VTE

Table 2 Inclusion and exclusion criteria for XALIA

\begin{tabular}{ll}
\hline Inclusion & Exclusion \\
\hline - Female or male patients who & $\begin{array}{l}\text { - Patients with acute } \\
\text { symptomatic PE* }\end{array}$ \\
$\begin{array}{ll}\text { - Diagnosis of acute DVT, } \\
\text { objectively confirmed }\end{array}$ & $\begin{array}{l}\text { - Other exclusion criteria are } \\
\text { dependent on local product } \\
\text { information }\end{array}$ \\
- Indication for anticoagulation & \\
therapy for at least 12 weeks & \\
- Patients willing to participate in the \\
study and available for follow-up
\end{tabular}

*A parallel symptomatic PE is not an exclusion criterion; however, XALIA will not enrol patients with only $P E$.

DVT, deep vein thrombosis; PE, pulmonary embolism. according to international guidelines, e.g. initial unfractionated heparin, LMWH or fondaparinux, which may overlap with and be followed by an oral VKA. However, in routine clinical practice many patients for whom rivaroxaban is planned may initially receive parenteral anticoagulants for a period longer than 48 hours before they are switched to rivaroxaban. These patients will be excluded from the main analysis, but the allocation of these patients will be subject to sensitivity tests as follows: 1) they will be analysed separately and 2) they will be allocated to standard of care or rivaroxaban dependent on the distribution of days (after the first 48 hours) in which they actually switched to rivaroxaban; the cut-off points that will be used for these sensitivity analyses will be defined by the quartiles of the distribution of days (after the 48 hours) in which the switch occurs.

Concomitant medications taken prior to or during the study will be documented and include non-steroidal anti-inflammatory drugs, acetylsalicylic acid, clopidogrel, steroids, medications known to interact with the DVT treatment, and medications known to increase or reduce the incidence of DVT (e.g. hormone therapy). Other drugs will be recorded if considered medically relevant by the investigator. Any changes to VTE treatment, including switching or cessation of treatment, will be documented.

\section{Data acquisition}

All patient data will be collected during the initial visit and routine follow-up visits (occurring around month 1 , quarterly and at final assessment) or by mail, telephone or email (Table 3). Demographic data and clinical characteristics will be collected from medical records if available, or by patient interviews. Patient-reported treatment satisfaction will be assessed using a specific treatment satisfaction questionnaire: the validated Anti-Clot Treatment Scale (ACTS), as used in the EINSTEIN DVT and EINSTEIN PE studies [24,25].

\section{Statistical analysis}

The primary statistical analysis includes a descriptive analysis of the primary and secondary outcomes. The primary outcome variable is the incidence of treatmentemergent adverse events, namely major bleeding and symptomatic recurrent VTE. An adverse event is considered as treatment emergent if it starts on or after the day of the first dose of study medication and $\leq 2$ days after the last dose. All patients who took at least one dose of medication will be included in the safety population. For primary and secondary variables, incidence proportions and incidence rates will be estimated, together with $95 \%$ confidence intervals. The accepted range for the $95 \%$ confidence intervals is estimated to be between the allocation ratios of $1: 3$ and $3: 1$; therefore, at least $25 \%$ of patients at each study centre 
Table 3 Overview of data collection procedures

\begin{tabular}{|c|c|c|c|c|}
\hline Scheduled procedure & $\begin{array}{l}\text { Initial visit } \\
\text { (baseline) }\end{array}$ & $\begin{array}{c}\text { After } \\
1 \text { month }\end{array}$ & Quarterly & Final assessment \\
\hline Visit date & $x$ & $x$ & $x$ & $x$ \\
\hline Demographic data & $x$ & & & \\
\hline Medical/surgical history & $x$ & & & \\
\hline Concomitant diseases & $x$ & & $X^{*}$ & $X^{*}$ \\
\hline Concomitant medication/treatment & $x$ & & $X^{*}$ & $X^{*}$ \\
\hline Physical examination & $x$ & & & \\
\hline Laboratory results (if available) & $x$ & $x$ & $x$ & $x$ \\
\hline Diagnosis of current DVT & $x$ & & & \\
\hline Previous VTE & $x$ & & & \\
\hline Current VTE treatment regimen & $x$ & & $x$ & $x$ \\
\hline Initial phase treatment & & $x$ & & \\
\hline Dose reduction (twice daily to once daily) & & $x$ & & \\
\hline Assessment of therapy & & & & $x$ \\
\hline Patient satisfaction and adherence with treatment (optional, only in a sub-sample of patients) & & $X^{*}$ & $X^{*}$ & $x^{*}$ \\
\hline Adverse events $^{\dagger}$ & & $x$ & $x$ & $x$ \\
\hline
\end{tabular}

${ }^{*}$ Only documented if new information is available from regular practice. No additional diagnostics are required for the study; ${ }^{\dagger}$ serious adverse events must be reported to the sponsor within 24 hours.

DVT, deep vein thrombosis; VTE, venous thromboembolism.

should receive either standard of care or rivaroxaban therapy. To compare the treatment arms, the risk difference and corresponding 95\% confidence interval will be calculated, and time-to-event analyses will be conducted.

The potential for allocation bias in open-label, nonrandomized studies will be minimized by also presenting a propensity score analysis to control the effect of unbalanced covariates [26,27]. To minimize selection bias, physicians will be asked to document patients consecutively. To decrease the possibility of reporting bias, source data will be verified at approximately $10 \%$ of study centres in countries where this is legally possible. A sample size of 4800 patients assumes that $17 \%$ of patients will be non-evaluable because of nonoverlapping propensity scores or because of withdrawn informed consent forms.

\section{Discussion}

The demand for real-world data in addition to clinical trial data is increasing. Non-interventional studies are crucial for obtaining a different perspective on the safety and effectiveness of drugs when used in routine clinical practice. Unlike registry data, which is typically analysed retrospectively, studies such as XALIA are designed to assess prospectively defined outcomes of interest thus eliminating potential bias. It is anticipated that XALIA will provide important information on the treatment of DVT in a heterogeneous patient population in a real-world setting. Strict inclusion and exclusion criteria in randomized clinical trials, such as EINSTEIN DVT, may restrict the population in which therapies can be studied, and it is important to show that the findings from clinical trials are applicable to patients treated in routine clinical practice.

One challenge with open-label observational studies is the potential for increased reporting of adverse events with the new drug, otherwise known as the Weber effect [28]. This may be a result of increased patient exposure and higher interest in the drug; however, when physicians become familiar with the drug and its adverse event profile, the rate of reported adverse events usually declines [28]. Therefore, it may be anticipated that this effect will also occur with rivaroxaban, at least during the initial period after its approval for the treatment of VTE.

Findings from XALIA may enable healthcare professionals to gain new insights into the management of patients with DVT, particularly regarding the risks of bleeding and recurrent VTE. Patients are at an immediate and continued risk of recurrence after the first venous thromboembolic event [29], and this risk can be substantial after cessation of anticoagulation [30,31]. Real-world data on recurrence rates will, therefore, be meaningful, particularly in patients who may discontinue anticoagulation. In some cases, the optimal duration of anticoagulation therapy for DVT is unclear; this may be partly owing to challenges in maintaining an appropriate benefit-risk balance because of an increased risk of bleeding or a patient's intrinsic risk factors [5]. It is hoped that by evaluating the incidence and type of thromboembolic 
events and bleeding events in XALIA, an accurate representation of the benefit-risk profile of rivaroxaban in routine care will be provided.

The clinical impact of anticoagulant-related bleeding complications is often severe. The case-fatality rate for major bleeding in patients with VTE has been reported to be as high as $11 \%$ within the first 3 months [32]. Furthermore, data from the RIETE registry showed that $56 \%$ of deaths in patients with VTE who received anticoagulation for 3 months were as a result of bleeding [33]. As mentioned previously, clinical trials are selective in nature, and may underestimate the frequency of bleeding seen in clinical practice. Results from a large community-based trial in the US showed an annual incidence of major bleeding as high as $12-13 \%$ in patients with VTE [34]. XALIA will provide valuable information on the incidence of major bleeding events that may occur with rivaroxaban or standard therapy in patients with DVT.

\section{Conclusions}

XALIA is a large, non-interventional study investigating the safety of rivaroxaban compared with standard of care for the treatment of acute DVT in routine clinical practice. Real-world data in a large cohort across multiple countries will be a valuable addition to the results of the EINSTEIN programme. The study was started in June 2012, will complete recruitment in early 2014, and results are expected in 2015.

\section{Competing interests}

WA has participated in advisory boards for Bayer HealthCare, Bristol-Myers Squibb (BMS), Pfizer and Daiichi Sankyo, and has received travel or research support from Bayer HealthCare, GlaxoSmithKline, Alexion Pharmaceuticals and Boehringer Ingelheim. LGM has been a consultant for Bayer HealthCare Pharmaceuticals and has received grants from Boehringer Ingelheim, Pfizer, BMS and Daiichi Sankyo. SH has been a consultant for Bayer HealthCare Pharmaceuticals, BMS, Boehringer Ingelheim, Daiichi Sankyo, Pfizer and Sanofi. RK has been a consultant for Bayer HealthCare, Berlin-Chemie, BMS and Daiichi Sankyo. AGGT has been a consultant for Bayer HealthCare, Janssen Pharmaceutical Research \& Development, LLC, Astellas, Portola and Takeda. VH and JS are both employees of Bayer HealthCare.

\section{Authors' contributions}

All authors contributed to the study concept, design and implementation, and to the content and development of this manuscript. All authors read and approved the final manuscript.

\section{Acknowledgements}

The XALIA study is sponsored by Bayer HealthCare Pharmaceuticals and Janssen Research \& Development, LLC. The authors would like to acknowledge David Whitford, who provided editorial support with funding from Bayer HealthCare Pharmaceuticals and Janssen Scientific Affairs, LLC.

\section{Author details}

'Department of Clinical and Experimental Medicine, University of Insubria, Via Guicciardini 9, Varese 21100, Italy. ${ }^{2}$ Department of Clinical Medicine and Surgery, Università degli Studi di Napoli Federico II, Naples, Italy. ${ }^{3}$ Vascular Centre, Normannenstr. 34a, Munich 81925, Germany. ${ }^{4}$ Institute of Clinical Pharmacology and Toxicology, Charité-Universitätsmedizin, Berlin, Germany. ${ }^{5}$ Bayer Vital GmbH, Leverkusen K56, 51368, Germany. ${ }^{6}$ Bayer Pharma AG,
Muellerstr. 178 S101, Berlin 13353, Germany. ${ }^{7}$ Department of Medicine, Hamilton Health Sciences, General Division, 237 Barton Street East, Hamilton, Ontario L8L 2X2, Canada.

Received: 11 February 2014 Accepted: 11 June 2014

Published: 14 July 2014

\section{References}

1. Goldhaber SZ: Venous thromboembolism: epidemiology and magnitude of the problem. Best Pract Res Clin Haematol 2012, 25:235-242.

2. Silverstein MD, Heit JA, Mohr DN, Petterson TM, O'Fallon WM, Melton $\sqcup$ III: Trends in the incidence of deep vein thrombosis and pulmonary embolism: a 25-year population-based study. Arch Intern Med 1998, 158:585-593.

3. Cushman M, Tsai AW, White RH, Heckbert SR, Rosamond WD, Enright $P$, Folsom AR: Deep vein thrombosis and pulmonary embolism in two cohorts: the longitudinal investigation of thromboembolism etiology. Am J Med 2004, 117:19-25.

4. Kearon C: A conceptual framework for two phases of anticoagulant treatment of venous thromboembolism. J Thromb Haemost 2012, 10:507-511.

5. Kearon C, AkI EA, Comerota AJ, Prandoni P, Bounameaux H, Goldhaber SZ, Nelson ME, Wells PS, Gould MK, Dentali F, Crowther M, Kahn SR: Antithrombotic therapy for VTE disease: antithrombotic therapy and prevention of thrombosis, 9th ed: American College of Chest Physicians evidence-based clinical practice guidelines. Chest 2012, 141:e419S-e494S.

6. Linkins LA, Dans AL, Moores LK, Bona R, Davidson BL, Schulman S, Crowther M: Treatment and prevention of heparin-induced thrombocytopenia: antithrombotic therapy and prevention of thrombosis, 9th ed: American College of Chest Physicians evidence-based clinical practice guidelines. Chest 2012, 141:e495S-e530S.

7. Ageno W, Gallus AS, Wittkowsky A, Crowther M, Hylek EM, Palareti G: Oral anticoagulant therapy: antithrombotic therapy and prevention of thrombosis, 9th ed: American College of Chest Physicians evidence-based clinical practice guidelines. Chest 2012, 141:e44S-e88S.

8. EINSTEIN The Investigators: Oral rivaroxaban for symptomatic venous thromboembolism. N Engl J Med 2010, 363:2499-2510.

9. The EINSTEIN-PE Investigators: Oral rivaroxaban for the treatment of symptomatic pulmonary embolism. N Engl J Med 2012, 366:1287-1297.

10. Agnelli G, Buller HR, Cohen A, Curto M, Gallus AS, Johnson M, Porcari A, Raskob GE, Weitz JI, AMPLIFY-EXT Investigators: Apixaban for extended treatment of venous thromboembolism. N Engl J Med 2013, 368:699-708.

11. Agnelli G, Buller HR, Cohen A, Curto M, Gallus AS, Johnson M, Masiukiewicz U, Pak R, Thompson J, Raskob GE, Weitz Jl, AMPLIFY Investigators: Oral apixaban for the treatment of acute venous thromboembolism. N Engl J Med 2013, 369:799-808.

12. Schulman S, Kearon C, Kakkar AK, Mismetti P, Schellong S, Eriksson H, Baanstra D, Schnee J, Goldhaber SZ, for the RE-COVER Study Group: Dabigatran versus warfarin in the treatment of acute venous thromboembolism. N Engl J Med 2009, 361:2342-2352.

13. Schulman S, Kearon C, Kakkar AK, Schellong S, Eriksson H, Baanstra D, Kvamme AM, Friedman J, Mismetti P, Goldhaber SZ: Extended use of dabigatran, warfarin, or placebo in venous thromboembolism. $N$ Engl J Med 2013, 368:709-718.

14. Schulman S, Kakkar AK, Goldhaber SZ, Schellong S, Eriksson H, Mismetti P, Vedel Christiansen A, Friedman J, Le Maulf F, Peter N, Kearon C, RE-COVER II Trial Investigators: Treatment of acute venous thromboembolism with dabigatran or warfarin and pooled analysis. Circulation 2014, 129:764-772.

15. The Hokusai-VTE Investigators: Edoxaban versus warfarin for the treatment of symptomatic venous thromboembolism. N Engl J Med 2013, 369:1406-1415.

16. Kubitza D, Becka M, Voith B, Zuehlsdorf M, Wensing G: Safety, pharmacodynamics, and pharmacokinetics of single doses of BAY 59-7939, an oral, direct Factor Xa inhibitor. Clin Pharmacol Ther 2005, 78:412-421.

17. Kubitza D, Becka M, Wensing G, Voith B, Zuehlsdorf M: Safety, pharmacodynamics, and pharmacokinetics of BAY 59-7939 - an oral, direct Factor Xa inhibitor - after multiple dosing in healthy male subjects. Eur J Clin Pharmacol 2005, 61:873-880.

18. Kubitza D, Becka M, Roth A, Mueck W: Dose-escalation study of the pharmacokinetics and pharmacodynamics of rivaroxaban in healthy elderly subjects. Curr Med Res Opin 2008, 24:2757-2765.

19. Kubitza D, Becka M, Schwers S, Voith B: Investigation of pharmacodynamic and pharmacokinetic interactions between rivaroxaban and enoxaparin in healthy male subjects. Clinical Pharm in Drug Dev 2013, 2:270-277. 
20. Kubitza D, Becka M, Mueck W, Zuehlsdorf M: Rivaroxaban (BAY 59-7939) - an oral, direct Factor Xa inhibitor - has no clinically relevant interaction with naproxen. Br J Clin Pharmacol 2007, 63:469-476.

21. Kubitza D, Becka M, Mueck W, Zuehlsdorf M: Safety, tolerability, pharmacodynamics, and pharmacokinetics of rivaroxaban - an oral, direct Factor Xa inhibitor - are not affected by aspirin. J Clin Pharmacol 2006, 46:981-990.

22. Kubitza D, Becka M, Roth A, Mueck W: Absence of clinically relevant interactions between rivaroxaban - an oral, direct Factor Xa inhibitor - and digoxin or atorvastatin in healthy subjects. J Int Med Res 2012, 40:1688-1707.

23. Stampfuss J, Kubitza D, Becka M, Mueck W: The effect of food on the absorption and pharmacokinetics of rivaroxaban. Int $J$ Clin Pharmacol Ther 2013, 51:549-561.

24. Bamber L, Wang MY, Prins MH, Ciniglio C, Bauersachs R, Lensing AWA, Cano SJ: Patient-reported treatment satisfaction with oral rivaroxaban versus standard therapy in the treatment of acute symptomatic deep-vein thrombosis. Thromb Haemost 2013, 110:732-741.

25. Prins M, Bamber L, Cano S, Wang M, Lensing A, Bauersachs R: Patient-reported treatment satisfaction with oral rivaroxaban versus standard therapy in the treatment of acute symptomatic pulmonary embolism [abstract]. Blood (ASH Annual Meeting Abstracts) 2012, 120. Abstract 1163.

26. Dahabreh IJ, Sheldrick RC, Paulus JK, Chung M, Varvarigou V, Jafri H, Rassen JA, Trikalinos TA, Kitsios GD: Do observational studies using propensity score methods agree with randomized trials? A systematic comparison of studies on acute coronary syndromes. Eur Heart J 2012, 33:1893-1901.

27. Collins GS, Le Manach Y: Comparing treatment effects between propensity scores and randomized controlled trials: improving conduct and reporting. Eur Heart J 2012, 33:1867-1869.

28. Weber JCP: Epidemiology of adverse reactions to nonsteroidal antiinflammatory drugs. In Advances in Inflammatory Research. Edited by Rainsford KD, Velo GP. New York: Raven Press; 1984:1-7.

29. Heit JA, Mohr DN, Silverstein MD, Petterson TM, O'Fallon WM, Melton LJ III: Predictors of recurrence after deep vein thrombosis and pulmonary embolism: a population-based cohort study. Arch Intern Med 2000 160:761-768.

30. Kearon C, Gent M, Hirsh J, Weitz J, Kovacs MJ, Anderson DR, Turpie AG, Green D, Ginsberg JS, Wells P, MacKinnon B, Julian JA: A comparison of three months of anticoagulation with extended anticoagulation for a first episode of idiopathic venous thromboembolism. N Engl J Med 1999, 340:901-907.

31. Chen SY, Wu N, Gulseth M, LaMori J, Bookhart BK, Boulanger L, Fields L, Schein J: One-year adherence to warfarin treatment for venous thromboembolism in high-risk patients and its association with long-term risk of recurrent events. J Manag Care Pharm 2013, 19:291-301.

32. Carrier M, Le Gal G, Wells PS, Rodger MA: Systematic review: case-fatality rates of recurrent venous thromboembolism and major bleeding events among patients treated for venous thromboembolism. Ann Intern Med 2010, 152:578-589.

33. Nieto JA, Camara T, Gonzalez-Higueras E, Ruiz-Gimenez N, Guijarro R, Marchena PJ, Monreal M: Clinical outcome of patients with major bleeding after venous thromboembolism. Findings from the RIETE Registry. Thromb Haemost 2008, 100:789-796.

34. Spencer FA, Gore JM, Reed G, Lessard D, Pacifico L, Emery C, Crowther MA, Goldberg RJ: Venous thromboembolism and bleeding in a community setting. The Worcester Venous Thromboembolism Study. Thromb Haemost 2009, 101:878-885.

\section{doi:10.1186/1477-9560-12-16}

Cite this article as: Ageno et al:: XALIA: rationale and design of a non-interventional study of rivaroxaban compared with standard therapy for initial and long-term anticoagulation in deep vein thrombosis. Thrombosis Journal 2014 12:16.

\section{Submit your next manuscript to BioMed Central and take full advantage of:}

- Convenient online submission

- Thorough peer review

- No space constraints or color figure charges

- Immediate publication on acceptance

- Inclusion in PubMed, CAS, Scopus and Google Scholar

- Research which is freely available for redistribution

Submit your manuscript at www.biomedcentral.com/submit 